Research Paper

\title{
Factors Influencing Clinicians' Choice of Adjuvant S-1 versus Capecitabine plus Oxaliplatin after Curative Gastrectomy in Patients with Gastric Cancer
}

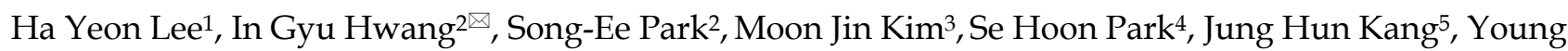
Saing Kim6, Sung Yong Oh7, Young-Woong Won ${ }^{8}$, Soon Il Lee ${ }^{9}$, Jun Ho Ji10, Kyong-Choun Chi11

1. Division of Hematology-Oncology, Department of Internal Medicine, Kyung Hee University Hospital at Gangdong, Seoul, South Korea

2. Department of Internal Medicine, Chung-Ang University College of Medicine, Seoul, South Korea

3. Division of Hematology-Oncology, Department of Medicine, Myongji Hospital, Goyang-si, Gyeonggido, South Korea

4. Division of Hematology-Oncology, Department of Medicine, Samsung Medical Center, Sungkyunkwan University School of Medicine, Seoul, South Korea

5. Division of Hematology-Oncology, Department of Internal Medicine, Gyeongsang National University Hospital, Gyeongsang National University School of Medicine, Jinju, South Korea

6. Division of Hematology and Oncology, Department of Internal Medicine, Gachon University Gil Medical Center, Incheon, South Korea

7. Department of Internal Medicine, Dong-A University College of Medicine, Busan, South Korea

8. Division of Hematology and Oncology, Department of Internal Medicine, Hanyang University College of Medicine, Seoul, South Korea

9. Division of Hematology-Oncology, Department of Internal Medicine, Dankook University College of Medicine, Cheonan, South Korea

10. Division of Hematology-Oncology, Department of Internal Medicine, Samsung Changwon Hospital, Sungkyunkwan University School of Medicine, Changwon, South Korea

11. Department of Surgery, Chung-Ang University College of Medicine, Seoul, South Korea

$\triangle$ Corresponding author: Division of Hematology/Oncology, Department of Internal Medicine, Chung-Ang University Hospital, Chung-Ang University College of Medicine, 102 Heukseok-ro, Dongjak-gu, Seoul 156-755, Republic of Korea. Tel: +82-2-6299-1403; Fax: 02-6299-1459; E-mail: hematoonco@naver.com

(C) Ivyspring International Publisher. Reproduction is permitted for personal, noncommercial use, provided that the article is in whole, unmodified, and properly cited. See http://ivyspring.com/terms for terms and conditions.

Received: 2016.03.21; Accepted: 2016.06.07; Published: 2016.07.27

\begin{abstract}
Purpose: Two recent randomized, phase III trials in Asia (ACTS-GC and CLASSIC) documented the survival benefit of postoperative chemotherapy after D2 lymph node dissection in patients with gastric cancer. We sought to determine what factors influenced clinicians' choices of either S-1 or capecitabine plus oxaliplatin (CAPOX) as adjuvant therapy after curative D2 gastrectomy.

Materials and Methods: We retrospectively reviewed the clinicopathologic factors and adjuvant treatments for 435 patients from nine centers in Korea who were treated with either S-1 or CAPOX adjuvant chemotherapy after undergoing curative D2 gastrectomy between January 2013 and July 2014.

Results: Of the 435 patients, 204 (46.9\%) were treated with S-1 and 231 (53.1\%) were treated with CAPOX. The median age at diagnosis was 61 years (range, 30-88). CAPOX was prescribed more often for patients who were 65 years of age or younger than for patients who were age 65 or older $(77.1 \%$ vs. $22.9 \%, \mathrm{P}<0.0001)$. Of the patients in stage II, $121(60.8 \%)$ were treated with $\mathrm{S}-1$ and $78(39.2 \%)$ were given CAPOX; however, of those in stage III, $83(35.2 \%)$ received S-1 and $153(64.8 \%)$ were treated with CAPOX $(\mathrm{P}<0.0001)$.

Conclusions: Clinicians only preferred CAPOX for younger patients with stage III gastric cancer after curative D2 gastrectomy. However, for elderly patients, clinicians more chose S-1 regardless of the stage.
\end{abstract}

Key words: stomach neoplasms, chemotherapy, adjuvant, gastrectomy

\section{Introduction}

Gastric cancer is the third leading cause of cancer death worldwide, with about 951,000 new cases and about 723,000 deaths occurring per year [1]. More than $70 \%$ of cases (about 677,000 cases) occur in developing countries, and half occur in East Asia [1]. Surgery is the only curative therapy for localized gastric cancer. However, in the majority of patients who undergo curative gastrectomy, the disease 
recurs.

Adjuvant therapy is a standard component of completely resected gastric cancer treatment. Two recent randomized, multicenter, phase III trials in Asia - the Adjuvant Chemotherapy Trial of TS-1 for Gastric Cancer (ACTS-GC) and the Adjuvant Capecitabine and Oxaliplatin for Gastric Cancer after D2 Gastrectomy trial (CLASSIC) - demonstrated a survival benefit with postoperative chemotherapy after D2 lymph node dissection in patients with gastric cancer [2-5]. Based on the results of these two studies, either of the two chemotherapy regimens used in these trials (i.e., S-1 or capecitabine plus oxaliplatin [CAPOX]) are considered to be effective in the treatment of gastric cancer after D2 dissection.

In selecting the appropriate regimen, clinicians take into account the patient's profile, the biology of the disease, and the potential impact of chemotherapy. In this study, we evaluated the clinicians' preferences and the criteria they applied when deciding between these two effective chemotherapeutic regimens.

\section{Materials \& Methods}

\section{Patients}

We conducted a retrospective analysis of the medical records of patients with advanced gastric cancer at nine institutes in Korea between January 2013 and July 2014. Patient characteristics at the time of diagnosis included age, sex, Eastern Cooperative Oncology Group (ECOG) performance status, and comorbidity. Clinicopathologic factors included date of surgery, type of surgery, histologic type, tumor stage, tumor grade, tumor location, and lymph node status. The treatment factors included the adjuvant chemotherapy regimen, the start and end dates of the adjuvant chemotherapy, number of cycles of adjuvant chemotherapy actually administered, the dose of chemotherapy actually given, the date of treatment was interrupted, date of disease progression, date of death, and date of last follow-up. Tumor histology and subtype were classified according to the World Health Organization criteria [6]. Tumor stage was classified according to the American Joint Committee on Cancer (AJCC) staging system (7th edition) [7]. The ECOG performance status was used to assess the functional status of each patient [8]. The study protocol was reviewed and approved by the institutional review boards of all participating centers.

\section{Treatments and assessments}

All patients received at least one cycle of adjuvant chemotherapy, either S-1 or CAPOX. The patients in the S-1 group were randomly assigned to receive S-1 (80 to $120 \mathrm{mg} /$ day) for 4 weeks, followed by 2 weeks of rest, and this 6-week cycle was repeated for 1 year. The patients in the CAPOX group were randomly assigned to receive eight 3-week cycles of oral capecitabine $\left(1,000 \mathrm{mg} / \mathrm{m}^{2}\right.$ twice daily on days 1 to 14 of each cycle) plus intravenous oxaliplatin (130 $\mathrm{mg} / \mathrm{m}^{2}$ on day 1 of each cycle). Tumor status was assessed by means of abdominal computed tomography or magnetic resonance imaging every 2 to 4 months while the patients were receiving the adjuvant chemotherapy and then every 3 to 6 months after the treatment had been completed.

\section{Statistical analysis}

The primary end point was the clinicians' preference for administering either adjuvant S-1 or adjuvant CAPOX after the patients had undergone curative gastrectomy. Categorical variables were compared with the use of the chi-square test, and continuous variables were analyzed by means of paired t-tests. $\mathrm{P}$ values less than 0.05 on all statistical tests were considered to be statistically significant. All analyses were performed with the SPSS Statistics, version 17.0 (SPSS Inc. Chicago, IL, USA).

\section{Results}

\section{Patient characteristics}

We retrospectively reviewed the records of 435 patients with gastric cancer who received adjuvant chemotherapy, either S-1 or CAPOX, after undergoing curative D2 gastrectomy between January 2013 and July 2014 at nine centers in Korea. Of the 435 patients, $204(46.9 \%)$ were treated with S-1 and 231 (53.1\%) were treated with CAPOX. The median age for the entire cohort at the time of diagnosis was 61 years (range, 30-88), and the median age was 66 years (range, 34-88) in the S-1 group and 58 years (range, 30-78) in the CAPOX group. There were 141 men in the S-1 group (69.1\%) and 145 men in the CAPOX group $(62.8 \%)$. The ECOG performance status (0-1) was better in the CAPOX group than in the S-1 group ( $97.8 \%$ vs. $90.2 \%, \mathrm{P}=0.002)$. The majority of tumors were located in the body and antrum of the stomach. Lymph node involvement was detected in $78.9 \%$ of the S-1 group and in $87.0 \%$ of the CAPOX group. The diagnosis was pathologic stage II in $60.8 \%$ of the S-1 group and in $39.2 \%$ of the CAPOX group, and pathologic stage III was the diagnosis in $35.2 \%$ of the S-1 group and in $64.8 \%$ of the CAPOX group. These results are summarized in Table 1.

\section{Chemotherapeutic regimen preferences}

In patients who were 65 years of age or younger, CAPOX was chosen more often than S-1 (65.9\% vs. $34.1 \%)$, whereas in those older than 65 years of age, 
S-1 was chosen more often than CAPOX $(67.9 \%$ vs. $32.1 \%)(\mathrm{P}<0.0001)$. In patients with pathologic stage T1-3 disease, S-1 was selected more often than CAPOX (54.9\% vs. $45.1 \%)$; however, in those with stage T4 disease, CAPOX was selected more often than S-1 $(68.7 \%$ vs. $31.3 \%)(\mathrm{P}<0.0001)$. For patients with nodal status 2 to 3,103 patients $(38.4 \%)$ received S-1 and 165 patients (61.6\%) received CAPOX. More of the patients with stage II disease were treated with S-1, and more of the patients with stage III disease received the CAPOX regimen $(\mathrm{P}<0.0001)$. The histologic grade was found to be poorly differentiated in $53.9 \%$ of the S-1 group and in $59.7 \%$ of the CAPOX group $(\mathrm{P}=0.369)$. There was no statistically significant difference in type of surgery between the two groups $(\mathrm{P}=0.235)$. These results are summarized in Table 2 .

Table 1. Patient characteristics

\begin{tabular}{|c|c|c|c|c|}
\hline \multicolumn{2}{|l|}{ Characteristic } & \multirow{2}{*}{ 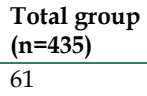 } & \multirow{2}{*}{$\begin{array}{l}\text { S-1 group } \\
(\mathbf{n}=\mathbf{2 0 4})\end{array}$} & \multirow{2}{*}{ 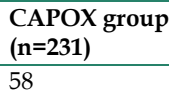 } \\
\hline Age, yr & Median & & & \\
\hline & Range & $30-88$ & $34-88$ & $30-78$ \\
\hline & $\leq 65$ & $270(62.1 \%)$ & $92(45.1 \%)$ & $178(77.1 \%)$ \\
\hline & $>65$ & $165(37.9 \%)$ & $112(54.9 \%)$ & $53(22.9 \%)$ \\
\hline Sex, n (\%) & Men & $286(65.7 \%)$ & $141(69.1 \%)$ & $145(62.8 \%)$ \\
\hline \multirow[t]{5}{*}{ ECOG, n $(\%)$} & 0 & $130(29.9 \%)$ & $69(33.8 \%)$ & $61(26.4 \%)$ \\
\hline & 1 & $280(64.4 \%)$ & $115(56.4 \%)$ & $165(71.4 \%)$ \\
\hline & 2 & $22(5.1 \%)$ & $17(8.3 \%)$ & $5(2.2 \%)$ \\
\hline & 3 & $1(0.2 \%)$ & $1(0.5 \%)$ & 0 \\
\hline & Unknown & $2(0.5 \%)$ & $2(1.0 \%)$ & 0 \\
\hline \multirow{8}{*}{$\begin{array}{l}\text { Tumor location, } \mathrm{n} \\
(\%)\end{array}$} & Antrum & $153(35.2 \%)$ & 78 (38.2\%) & $75(32.5 \%)$ \\
\hline & Body & $200(46.0 \%)$ & $75(36.7 \%)$ & $125(54.1 \%)$ \\
\hline & $\begin{array}{l}\text { Body and } \\
\text { antrum }\end{array}$ & $42(9.7 \%)$ & $30(14.7 \%)$ & $12(5.2 \%)$ \\
\hline & Fundus & $3(0.7 \%)$ & $2(1.0 \%)$ & $1(0.4 \%)$ \\
\hline & $\begin{array}{l}\text { Fundus and } \\
\text { body }\end{array}$ & $13(3.0 \%)$ & $5(2.5 \%)$ & $8(3.5 \%)$ \\
\hline & GE junction & $10(2.3 \%)$ & $6(3.0 \%)$ & $4(1.7 \%)$ \\
\hline & Whole gastric & $10(2.3 \%)$ & $5(2.5 \%)$ & $5(2.2 \%)$ \\
\hline & Othera) & $4(0.9 \%)$ & $3(1.5 \%)$ & $1(0.4 \%)$ \\
\hline \multirow{5}{*}{$\begin{array}{l}\text { Tumor stage, } \mathrm{n} \\
(\%)\end{array}$} & $\mathrm{T} 1$ & $29(6.7 \%)$ & $17(8.3 \%)$ & $12(5.2 \%)$ \\
\hline & $\mathrm{T} 2$ & $61(14.0 \%)$ & $31(15.2 \%)$ & $30(13.0 \%)$ \\
\hline & T3 & $198(45.5 \%)$ & $110(53.9 \%)$ & $88(38.0 \%)$ \\
\hline & $\mathrm{T} 4 \mathrm{a}$ & $136(31.3 \%)$ & $40(19.6 \%)$ & $96(41.6 \%)$ \\
\hline & $\mathrm{T} 4 \mathrm{~b}$ & $11(2.5 \%)$ & $6(3.0 \%)$ & $5(2.2 \%)$ \\
\hline \multirow{4}{*}{$\begin{array}{l}\text { Nodal status, } \mathrm{n} \\
(\%)\end{array}$} & N0 & $73(16.8 \%)$ & $43(21.1 \%)$ & $30(13.0 \%)$ \\
\hline & N1 & $94(21.6 \%)$ & $58(28.4 \%)$ & $36(15.6 \%)$ \\
\hline & N2 & $115(26.4 \%)$ & $47(23.0 \%)$ & $68(29.4 \%)$ \\
\hline & N3 & $153(35.2 \%)$ & $56(27.5 \%)$ & 97 (42.0\%) \\
\hline \multirow[t]{5}{*}{ AJCC stage, n (\%) } & IIA & $98(22.5 \%)$ & $65(31.9 \%)$ & $33(14.3 \%)$ \\
\hline & IIB & $101(23.2 \%)$ & $56(27.4 \%)$ & $45(19.5 \%)$ \\
\hline & IIIA & $72(16.6 \%)$ & $26(12.7 \%)$ & $46(19.9 \%)$ \\
\hline & IIIB & $84(19.3 \%)$ & $33(16.2 \%)$ & $51(22.1 \%)$ \\
\hline & IIIC & $80(18.4 \%)$ & $24(11.8 \%)$ & $56(24.2 \%)$ \\
\hline
\end{tabular}

a) Other locations were body plus cardia and fundus, body plus gastroesophageal (GE) junction

AJCC, American Joint Committee on Cancer; CAPOX, capecitabine plus oxaliplatin; ECOG, Eastern Cooperative Oncology Group.
Relatively more patients with stage II disease who were older than 65 years of age were given S-1 than CAPOX $(77.0 \%$ vs. $23.0 \%)$; however, of those patients who had stage II disease and who were 65 years of age or younger, the percentage of patients assigned to S-1 or CAPOX was similar $(51.2 \%$ vs. $48.8 \%$ ). For patients with stage III disease, more patients who were age 65 or younger received CAPOX $(80.7 \%)$ than received S-1 $(19.3 \%)$, whereas more patients who were older than age 65 were treated with S-1 (60.4\%) than with CAPOX (39.6\%) $(\mathrm{P}<0.0001)$. There were significant differences in the adjuvant chemotherapy regimen according to age in the patients with stage III disease $(\mathrm{P}<0.0001)$. In addition, more patients with stage II disease received S-1 than received CAPOX irrespective of nodal status $(\mathrm{P}=0.959)$. These results are summarized in Table 3.

Table 2. Subgroup analysis according to patient characteristics

\begin{tabular}{|c|c|c|c|c|}
\hline Characteristic & & $\begin{array}{l}S-1 \text { group } \\
(n=204)\end{array}$ & $\begin{array}{l}\text { CAPOX group } \\
(n=231)\end{array}$ & $P$ value \\
\hline \multirow[t]{2}{*}{ Age, n (\%) } & $\leq 65 \mathrm{yr}$ & $92(34.1 \%)$ & $178(65.9 \%)$ & $<0.0001$ \\
\hline & $>65 \mathrm{yr}$ & $112(67.9 \%)$ & $53(32.1 \%)$ & \\
\hline \multirow{2}{*}{$\begin{array}{l}\text { Tumor stage, } \mathrm{n} \\
(\%)\end{array}$} & $\mathrm{T} 1-3$ & $158(54.9 \%)$ & $130(45.1 \%)$ & $<0.0001$ \\
\hline & $\mathrm{T} 4$ & $46(31.3 \%)$ & $101(68.7 \%)$ & \\
\hline \multirow{2}{*}{$\begin{array}{l}\text { Nodal status, n } \\
(\%)\end{array}$} & N0-1 & $101(60.5 \%)$ & $66(39.5 \%)$ & $<0.0001$ \\
\hline & $\mathrm{N} 2-3$ & $103(38.4 \%)$ & $165(61.6 \%)$ & \\
\hline \multirow[t]{2}{*}{ AJCC stage, $\mathrm{n}(\%)$} & II & $121(60.8 \%)$ & $78(39.2 \%)$ & $<0.0001$ \\
\hline & III & $83(35.2 \%)$ & $153(64.8 \%)$ & \\
\hline \multirow[t]{2}{*}{ Surgery } & Total & $70(43.2 \%)$ & $92(56.8 \%)$ & 0.235 \\
\hline & Subtotal & $134(49.1 \%)$ & $139(50.9 \%)$ & \\
\hline
\end{tabular}

AJCC, American Joint Committee on Cancer; CAPOX, capecitabine plus oxaliplatin

Table 3. Subgroup analysis according to age and stage

\begin{tabular}{lllll}
\hline \multicolumn{1}{l}{ Characteristic } & S-1 (n=204) & CAPOX (n=231) & P value \\
\hline Age $\leq 65$ & Stage II & $64(51.2 \%)$ & $61(48.8 \%)$ & $<0.0001$ \\
& Stage III & $28(19.3 \%)$ & $117(80.7 \%)$ & \\
\multirow{2}{*}{ Age $>65$} & Stage II & $57(77.0 \%)$ & $17(23.0 \%)$ & 0.023 \\
& Stage III & $55(60.4 \%)$ & $36(39.6 \%)$ & \\
Stage II & Age $\leq 65$ & $64(51.2 \%)$ & $61(48.8 \%)$ & $<0.0001$ \\
& Age $>65$ & $57(77.0 \%)$ & $17(23.0 \%)$ & \\
Stage III & Age $\leq 65$ & $28(19.3 \%)$ & $117(80.7 \%)$ & $<0.0001$ \\
& Age $>65$ & $55(60.4 \%)$ & $36(39.6 \%)$ & \\
\multirow{2}{*}{ Stage II } & Node - & $43(60.6 \%)$ & $28(39.4 \%)$ & 0.959 \\
& Node + & $78(60.9 \%)$ & $50(39.1 \%)$ & \\
& & &
\end{tabular}

\section{Discussion}

Because no clinical trials have directly compared the use of S-1 versus CAPOX, in this study we evaluated the trend among clinicians in their choice of either S-1 or CAPOX as adjuvant therapy after curative D2 gastrectomy. We found that clinicians tended to choose S- 1 for elderly patients but tended to select CAPOX for younger patients with a more advanced stage of disease. Age was therefore an 
important factor in the selection of an adjuvant chemotherapy regimen. The patients of age 65 or younger were significantly more likely to receive CAPOX $(65.9 \%)$, but the patients older than age 65 were more likely to be treated with S-1 (67.9\%).

In the CLASSIC study, 5-year disease-free survival (DFS) in the adjuvant CAPOX group was significantly better than in the surgery-alone group among patients who were younger than age 65 and older than age 65 [5]. In addition, 5-year overall survival (OS) in the adjuvant CAPOX group was significantly better than in the surgery-alone group in the patients who were younger than age 65; however, there were no differences in 5-year OS between the CAPOX group and the surgery-alone group in the patients older than age 65. In the ACTS-GC trial, patients who were younger than age 60 had significantly better 5-year relapse-free survival (RFS) and 5-year OS with S-1 when compared with the surgery-alone group [3]. Patients who were younger than age 69 had significantly better 5-year OS with S-1, but 5-year OS did not differ significantly in the patients who were age 70 or older with S-1 when compared with the surgery-alone group.

Our study showed that clinicians more often selected CAPOX for patients who were age 65 or younger. In the ACTS-GC trial, adjuvant S-1 resulted in better 5-year OS in the patients who were younger than age 69 , but there were no significant differences in 5-year OS between this group and those who were age 70 or older [3]. In addition, there were no significant differences in 5-year RFS between the S-1 group and the surgery-alone group among the patients who were older than age 60. Based on these results, it might be that the clinicians more often chose S-1 for the patients who were older than age 65 owing to the reported toxicity of CAPOX. In the CLASSIC trial, the most commonly reported adverse events of any grade in the CAPOX group were nausea $(66 \%)$, neutropenia $(60 \%)$, decreased appetite $(59 \%)$, and peripheral neuropathy (56\%) [5]. The most common grade 3 or 4 adverse events in the CAPOX group were neutropenia $(22 \%)$, nausea $(8 \%)$, thrombocytopenia (8\%), and vomiting (7\%) [5]. Therefore 33.5\% $(174 / 520)$ did not complete the CAPOX regimen. However, in the ACTS-GC trial, grade 3 or 4 adverse events occurred in less than $5 \%$ of the S-1 group [2].

Our study showed that more patients with nodal status 2-3 received CAPOX than received S-1, but more patients with nodal status $0-1$ were treated with S-1 than with CAPOX. In the CLASSIC trial, the chemotherapy group had significantly better 5-year DFS and OS than did the surgery-alone group in the patients with node-positive gastric cancer [5]. However, in the ACTS-GC trial, the S-1 group had significantly better 5-year DFS and OS than did the surgery-alone group in the patients with nodal status $0-1$, but in the patients with nodal status 2 , there was no significant difference in 5-year DFS and OS between the S-1 group and the surgery-alone group [3]. These results might influence clinicians when selecting an adjuvant chemotherapy regimen.

Our results demonstrated that more patients with stage II disease were treated with S-1 and more patients with stage III disease were treated with CAPOX. In the CLASSIC trial, the CAPOX regimen resulted in significantly better 5-year DFS and OS as compared with surgery alone in the patients with stage II disease [5]. However, in the patients with stage III disease, 5-year DFS was significantly better in the CAPOX group, but there was no difference in 5-year OS between the CAPOX and surgery-alone groups. In the ACTS-GC trial, S-1 also resulted in significantly better 5-year DFS and OS as compared with surgery alone in the patients with stage II disease [3]. But in the patients with stage III disease, there was no significant difference in 5-year RFS and OS between the S-1 group and the surgery-alone group. Despite the results of these two trials, the clinicians thought that a more intensive chemotherapy might be effective in the patients with advanced disease.

Relatively more patients with stage II disease who were older than 65 years of age were treated with S-1 than with CAPOX (77.0\% vs. $23.0 \%$ ). However, the percentage of patients age 65 or younger with stage II disease who received S-1 and those who received CAPOX was similar (51.2\% and $48.8 \%$, respectively). Relatively more patients with stage III disease who were age 65 or younger were treated with CAPOX than with S-1 (80.7\% vs. 19.3\%). Furthermore, more patients who were older than age 65 with stage III disease received CAPOX than received S-1 (60.4\% vs. $39.6 \%)$.

It was reasonable that more patients who were older and in stage II received S-1 than received CAPOX and that more patients who were younger and in stage III were treated with CAPOX than with S-1. However, when there were conflicts with respect to patient factors and disease factors - in other words, patients who were younger and in stage II similar percentages of patients were treated with S-1 or CAPOX. Although the two previously reported studies showed that both these chemotherapy regimens significantly improved 5-year DFS and OS, as compared with surgery alone, in the younger patients, our study indicated that clinicians similarly chose either S-1 or CAPOX in younger patients with early-stage disease.

In conclusion, our results show that clinicians preferred S-1 as adjuvant treatment for patients who 
were elderly or had early-stage gastric cancer. However CAPOX were highly selected for patients who were younger and had more advanced disease. We are continuing follow-up this study cohort and are collecting data for compliance and survival until now. We will report the relationship of survival according to preference of clinicians for this study population after long-term follow-up.

\section{Competing Interests}

The authors have declared that no competing interest exists.

\section{References}

1. Ferlay J, Soerjomataram I, Dikshit R, Eser S, Mathers C, Rebelo M, et al. Cancer incidence and mortality worldwide: sources, methods and major patterns in GLOBOCAN 2012. International journal of cancer Journal international du cancer. 2015; 136: E359-86.

2. Sakuramoto S, Sasako M, Yamaguchi T, Kinoshita T, Fujii M, Nashimoto A, et al. Adjuvant chemotherapy for gastric cancer with S-1, an oral fluoropyrimidine. N Engl J Med. 2007; 357: 1810-20.

3. Sasako M, Sakuramoto S, Katai H, Kinoshita T, Furukawa H, Yamaguchi T, et al. Five-year outcomes of a randomized phase III trial comparing adjuvant chemotherapy with S-1 versus surgery alone in stage II or III gastric cancer. J Clin Oncol. 2011; 29: 4387-93.

4. Bang YJ, Kim YW, Yang HK, Chung HC, Park YK, Lee KH, et al. Adjuvant capecitabine and oxaliplatin for gastric cancer after D2 gastrectomy (CLASSIC): a phase 3 open-label, randomised controlled trial. Lancet. 2012; 379: 315-21.

5. Noh SH, Park SR, Yang HK, Chung HC, Chung IJ, Kim SW, et al. Adjuvant capecitabine plus oxaliplatin for gastric cancer after D2 gastrectomy (CLASSIC): 5-year follow-up of an open-label, randomised phase 3 trial. The Lancet Oncology. 2014; 15: 1389-96.

6. Fenoglio-Preiser C AF, Correa $\mathrm{P}$, et al. World Health Organization classification of tumours. Pathology and genetics of tumours of the digestive system. Lyon: IARC. 2000.

7. Edge SB, Compton CC. The American Joint Committee on Cancer: the 7th edition of the AJCC cancer staging manual and the future of TNM. Annals of surgical oncology. 2010; 17: 1471-4.

8. Oken MM, Creech RH, Tormey DC, Horton J, Davis TE, McFadden ET, et al. Toxicity and response criteria of the Eastern Cooperative Oncology Group. American journal of clinical oncology. 1982; 5: 649-55.

9. Koizumi W, Narahara H, Hara T, Takagane A, Akiya T, Takagi M, et al. S-1 plus cisplatin versus S-1 alone for first-line treatment of advanced gastric cancer (SPIRITS trial): a phase III trial. The Lancet Oncology. 2008; 9: 215-21. 